\title{
Comparison of Graph-based and Hypergraph-based Models for Wireless Network Coexistence
}

\author{
$1^{\text {st }}$ Tawachi Nyasulu \\ Department of Electronic and Electrical Engineering \\ University of Strathclyde \\ Glasgow, United Kingdom \\ Email: tawachi.nyasulu@strath.ac.uk
}

\author{
$2^{\text {nd }}$ David H. Crawford \\ Department of Electronic and Electrical Engineering \\ University of Strathclyde \\ Glasgow, United Kingdom \\ Email: david.crawford@strath.ac.uk
}

\begin{abstract}
Dynamic Spectrum Access (DSA) is regarded as a promising solution for efficient spectrum management. Regulators have also approved licence-exemption or general authorisation access (GAA) to further improve spectrum accessibility for DSA systems in the Television (TV) and $3.5 \mathrm{GHz}$ bands. However, heterogeneous DSA radio standards have been developed and the gains in spectrum efficiency could be undermined by coexistence challenges. Hence, the IEEE 802.19.1 standard for wireless network coexistence methods was published, but it leaves algorithmic implementation of the methods to the industry. When the spectrum is not sufficient for exclusive channel allocation, the standard includes a method for co-channel sharing among coexistent neighbour networks. In previous work, channel sharing was introduced on top of the exclusive channel allocation. However, channel sharing options could be significantly limited by the outcome of the exclusive channel allocation. Alternatively, this paper proposes use of hypergraph theory to model the cosharing strategy for coexistence management of heterogeneous radio systems. Results demonstrate that the hypergraph method achieves higher average spectrum utilisation by up to $8 \%$ and requires up to 5 fewer channels to achieve, on average, $100 \%$ operational networks than the previous method.
\end{abstract}

\section{INTRODUCTION}

Accessibility of radio frequency (RF) spectrum is a critical factor in the growth of wireless networks. Dynamic Spectrum Access (DSA) enables secondary users to access the spectrum of licensed (primary) users on licence-exemption or on temporary exclusive rights bases, when the spectrum is not being used by the primary users. The US and the UK have approved licence-exemption or GAA policy for DSA systems in Television White Spaces (TVWS). The US has also approved GAA as one of the access policies in the 3.5 $\mathrm{GHz}$ Citizen Broadband Radio Service (CBRS). The main motivation for licence fee exemption is to stimulate innovation and business start-ups.

GAA users are not guaranteed any interference protection. Licence-exempt spectrum may be prone to overuse and can potentially result in performance degradation as a result of interference amongst secondary users. Cognitive radios which have been developed for operation in DSA spectrum are capable of spectrum mobility in response to the conditions in the RF environment. However, in the presence of multiple independent heterogeneous networks and without coordinated spectrum allocation, there is a challenge related to switching between available channels to achieve coexistence and efficient spectrum usage, when the amount of available spectrum is not sufficient for each network to operate exclusively in a channel [1], [2]. The gains in spectrum accessibility as a result of licence-exemption policy can potentially be diminished by the impact of interference.

Coexistence mechanisms for heterogeneous wireless protocols, targeted at the media access control/physical (MAC/PHY) layers, have been proposed [3]. However, this approach requires modifications to the radio protocols and hardware and may not be sustainable as radio technology standards evolve and as new standards are introduced. The IEEE 802.19.1 standard therefore specifies high level, radiotechnology-independent, network-based coexistence methods instead. However, actual implementation of the algorithms is left to the industry.

This paper, while building on the methods proposed in the IEEE 802.19.1 standard and on previous work, proposes use of hypergraph theory to represent potential interference and spectral coexistence relationships among independent heterogeneous networks in order to implement coordinated channel allocation and coexistence management. The model can be applied in IEEE 802.19.1-based centralised coexistence management systems. The performance of the proposed solution is compared with previous work in [4].

The paper is organised as follows. Literature is analysed in Section II. The system design is described in Section III. The graph-based method from previous work is reproduced in Section IV. The proposed hypergraph-based method is described in Section V. Simulation results are discussed in Section VI. Finally, conclusions are drawn in Section VII.

\section{Analysis of Previous Work}

Graph colouring is conventionally used to model channel assignment in space and frequency domain [2], [4], [5]. Spatial re-usability of the available spectrum could be improved through transmission power control. Spectral efficiency could also be improved through co-sharing in the time-domain between networks that can coexist in the same spectrum using compatible media access control mechanisms [2].

In [6], spectral coexistence relationships between a pair of networks was represented by a super nodes in a coexistence- 
aware conflict subgraph. When a super node is added to a conflict graph, it inherits conflict relations of its children nodes and the edge between its children pairs is removed. Thus, super nodes have a high number of incident edges, giving the channel assignment algorithm too many paths to consider.

A coexistence decision algorithm for spectrum sharing is proposed in [4] for use in IEEE 802.19.1 systems. The algorithm introduces shared spectrum allocation on top of exclusive channel allocation. The algorithm selects a network at each step and assigns spectrum to the network. If no unoccupied channel is available, the algorithm searches for a channel occupied by neighbour networks of the same MAC/PHY type, subject to channel load constraints. While this approach ensures spectrum allocation stability in that previous allocations are not rearranged to accommodate new networks, channel sharing options are dependent on the previous exclusive channel allocations. A more efficient channel allocation could be realised if the previous allocations could be re-arranged. Algorithms that minimise the need and frequency to change channel assignment can also be applied.

The RF environment could be organised into coexistent groups so that channels are allocated to groups, instead of individual networks. But, the concept of an edge in a traditional graph, which is a two-element subset, is not sufficient to model subsets of potential co-sharing networks because such subsets may have cardinality of greater than 2. This paper proposes hypergraph theory as an alternative mathematical tool for representing groups of coexistent networks so that the channel allocation algorithm is able to assign channels to groups of coexistent networks instead of individual networks. A hypergraph is a generalization of an undirected graph in which a hyperedge is a subset of vertices of arbitrary cardinality rather than two-element subsets [7]. Hypergraphs have found application in modelling of cumulative interference [8] and network dependency [9]. To the best of our knowledge, hyperedges have not been used before to model spectral coexistence among multiple independent networks for spectrum sharing.

\section{System Design}

The test scenario consists of IEEE 802.22 wireless regional area networks (WRANs) and 802.11 television very high throughput (TVHT) wireless local area networks (WLANs). The base station (BS) and access point (AP) radios support a set of slave devices in the form of customer premise equipment (CPEs) and user devices (UEs), respectively. Each master device and its slave devices communicate on the same channel and the master device coordinates access to the channel among its slave devices. Information about the geo-location, service area, receiver sensitivity and RAT of each master device is known. The service/coverage area of a network is specified by its coverage radius with reference to the location of the master device and its slave devices are located within the coverage area.

\section{A. Interference Analysis}

Only co-channel interference among secondary user networks is considered. Primary users are protected by a Geolocation Spectrum Database (GLDB). Adjacent Channel Leakage Ratio (ACLR) performance of TVWS radios is regulated in order to prevent interference to licensed primary users. It is assumed that all networks have overlap in their operating frequency capabilities. Interference levels are calculated for the worst case scenario when the master device is transmitting at maximum power permissible by regulation or attainable by the RAT standard, and at the lowest frequency where TVWS is permitted in the TV band.

Only interference caused or received by the BS/AP radio is considered for signal-to-interference-plus-noise-ratio (SINR) constraint because it usually transmits at a higher power using an omni-directional antenna and it is therefore more likely to cause or receive interference than the CPE/UE radio. Even though CPEs use directional antennas, CPEs that are within the line of sight of more than one BS will suffer or cause interference. It is assumed that this scenario will be avoided at the time of CPE installation since the IEEE 802.22.2 Standard for Installation and Deployment of IEEE 802.22 Systems specifies that the CPE antenna should be oriented toward its serving BS and should be further adjusted to minimise the gain in the direction of an interfering $\mathrm{BS}$ while keeping the gain toward its serving BS within $2 \mathrm{~dB}$ of its maximum.

Consider two cells, $i$ and $j$, with master devices $W_{i}$ and $W_{j}$, and with coverage radii $r_{i}$ and $r_{j}$. Let $d_{i j}$ be the physical distance between the two master devices that is calculated from their geo-locations using the Haversine formula. A pair of networks are neighbours if their coverage areas overlap and this is evaluated by eq. (1).

$$
d_{i j}<r_{i}+r_{j}
$$

If the coverage areas of two networks do not overlap, as illustrated in Fig. 1, these are neighbours if the SINR of celledge users, which experience lowest SINR due to their distant locations, is less than a set threshold, $\delta$. This condition is satisfied if eq. (2) and/or eq. (3) are/is true.

$$
\begin{aligned}
& \frac{P_{i}}{P_{j}}<\delta_{i} ; \text { at the edge of network } i \\
& \frac{P_{j}}{P_{i}}<\delta_{j} ; \text { at the edge of network } j
\end{aligned}
$$

Interference information is represented by an undirected graph $G=(V, E)$ using adjacency lists. Construction of the interference graph is described in Algorithm 1.

\section{B. Spectral Coexistence Analysis}

Multiple IEEE WRAN BSs use on-demand frame contention to share a channel in the time domain. Timesynchronisation can happen over wireless connection if the network controllers are within each other's communication range. In WLANs, UEs use CSMA/CA with Request to 


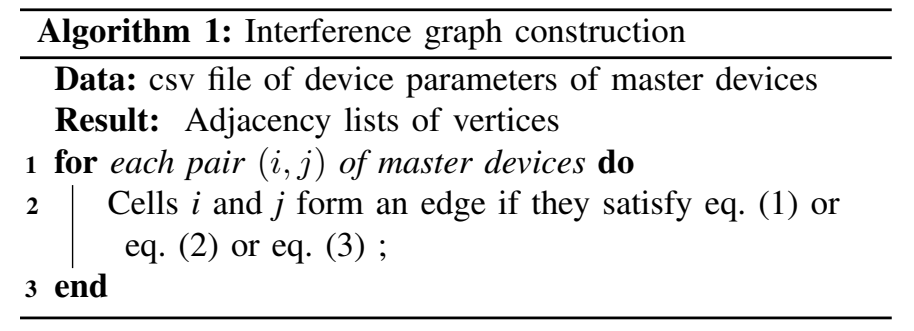

Send/Clear To Send (RTS/CTS) to minimise collisions, as specified in the IEEE 1900.7 standard for white space DSA radio systems. While the IEEE 802.11 TVHT devices can back-off when an IEEE 802.22 radio is transmitting, the IEEE 802.22 would not back off because it is an 'impolite' radio. Thus, the two standards cannot effectively coexist in the same channel.

The method for evaluation of coexistence is based on the IEEE 802.19.1 mechanisms for "co-sharing via network geometry classification", and two classes in which synchronisation happens over the wireless connection are considered.

a) The coverage area of a smaller network is completely overlaid by that of a wider network (Class 4): In such cases, the two networks can share a channel if they communicate using compatible RATs and if the interference power from the wider network to the smaller network is not harmful. BS interference into neighbouring BSs can be prevented by avoiding line of sight and/or maximising the separation distances between BSs. It is assumed that line of sight would be avoided at the time of installation. The absolute minimum separation between antennas must be a horizontal distance of greater than $1 / 4$ of its wavelength, but it should not be located at the exact multiples of its wavelength. The longest wavelength in the TV band is $0.63247 \mathrm{~m}$ for the centre frequency of the lowest channel, $474 \mathrm{MHz}$. In [10], a controlled study was conducted to analyse performance and coexistence among IEEE 802.15.4, 802.11 and 802.22 radio systems. The study concluded that generally, all of the systems would significantly deteriorate if the interferer is located within $12 \mathrm{~m}$. Hence, a separation distance of $15 \mathrm{~m}$ is used in this model. Thus, networks $i$ and $j$ are considered as coexisting networks if (4) is satisfied.

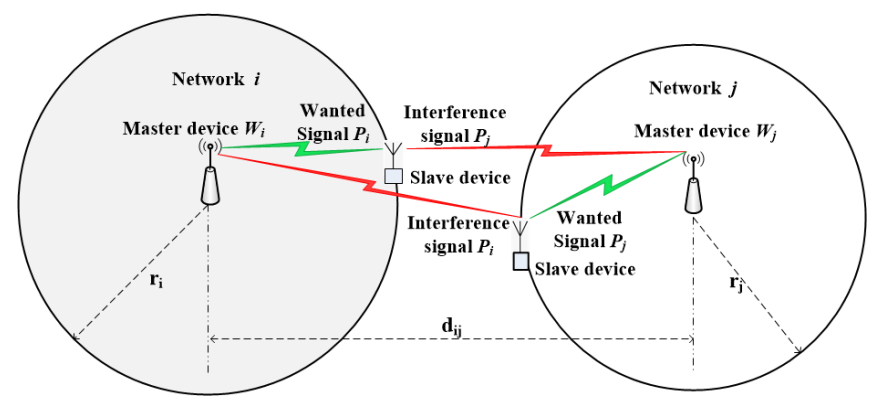

Fig. 1: Interference analysis between a pair of networks with non-overlapping coverage areas.

$$
d_{i j}<r_{i} \text { or } d_{i j}<r_{j} \text { and } d_{i j}>15 m
$$

b) The coverage areas of $W_{i}$ and $W_{j}$ overlap and the two master devices are within each other's communication range (Class 1): This class is satisfied if equations (1), (5) and (6) hold true. The signal-to-noise-ratio (SNR) is calculated at the geo-location of the other master device. The noise floor, $N$, is based on the receiver sensitivity. The SNR must be greater than a set threshold, $\delta$, for the master devices to be able to communicate.

$$
\begin{aligned}
& \frac{P_{i}}{N}>\delta_{j} ; \text { at the geo-location of master device } W_{j} \\
& \frac{P_{j}}{N}>\delta_{i} ; \text { at the geo-location of master device } W_{i}
\end{aligned}
$$

Spectral coexistence information is stored in an $N \times N$ matrix $\mathrm{C}$, where an entry of 1 for $C_{i j}$ signifies that networks $i$ and $j$ can coexist.

\section{Performance Metric}

Given a set of $N$ networks that are competing for the same channels and a set of $M$ channels, assuming that all channels are available at the geo-locations of all the networks, channel allocation is represented by a matrix $R_{N \times M}=\left[\beta_{n, m}\right]$, where $\beta_{n, m}=1$ when channel $m$ is allocated to network $n$; 0 otherwise. The number of operational networks $\alpha$, having been assigned an operating channel from a set of available channels, is expressed as: $\alpha=\sum_{m=1}^{M} \sum_{n=1}^{N} \beta_{n, m}$. The performance metric is the average number of operational networks from $K$ number of simulations, which is given by: $\alpha_{\text {avg }}=\left(\sum_{k=1}^{K}\right) / K$, which is also expressed as a percentage of the total number of competing networks, $N$.

\section{Graph-Based Spectrum Sharing Model}

In this section, the method that was proposed in [4] is reproduced. Radio resource allocation is solved using a vertex colouring algorithm whereby a vertex represents a cell and a colour assignment represents a channel allocation. The vertex colouring problem seeks to colour the vertices using the minimum number of colours such that all neighbour vertices are coloured using different colours. Thus, a cell is assigned an exclusive channel among its neighbour cells. Nodes are coloured in descending order of vertex degree since high degree vertices represent the networks that interfere with more networks and therefore have more colour constraints. Where there is a tie, the vertices are coloured in ascending order of network identity number. Thereafter, channel sharing is applied so that if a network could not be allocated an exclusive channel, it can share the same channel with networks of the same RAT that have already been allocated the channel, on the conditions that it can coexist with all other neighbour networks that have already been assigned that channel and that the total channel occupancy rate (COR) would not exceed unity when the new network joins the channel. The entire procedure is summarised in Algorithm 2. 


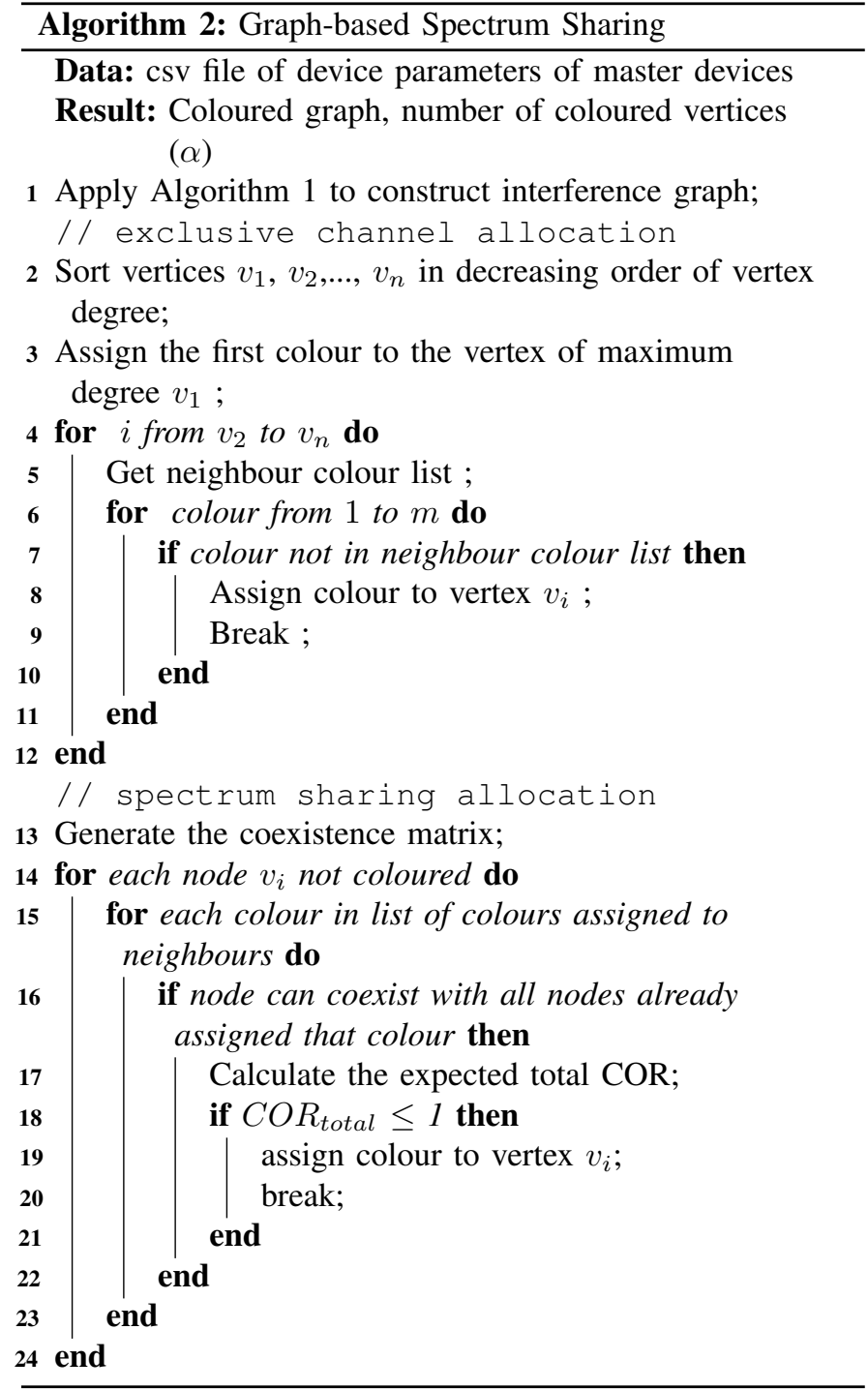

\section{Hypergraph-Based Spectrum Sharing Model}

In hypergraphs, hyperedges represent subsets of any cardinality, not just 2 as in graphs, such that hyperedges can be used to represent "arbitrary general statements about arbitrary subsets" [7]. Let $H=(X, E)$ be a hypergraph. $X=\left\{x_{1}, x_{2}, \ldots, x_{n}\right\}$ is a set of the vertices and $E=\left\{e_{1}, e_{2}, \ldots, e_{m}\right\}$ is a set of hyperedges of hypergraph $H$ such that $e_{j} \neq \emptyset$ and $\bigcup_{i=1}^{m} e_{j}=X$.

A hypergraph is represented in the form of an incidence matrix, $I(H)$. A vertex $x_{i} \in X$ and a hyperedge $e_{j} \in E$ are said to be incident to each other if $x_{i}$ belongs to hyperedge $e_{j}$. Any two hyperedges are said to be adjacent if the intersection of their sets is not an empty set. Weak deletion of $e_{j} \in E$ from $H$ is to remove $e_{j}$ from $E$. Contraction of hyperedges is widely used in many algorithms to decompose a hypergraph [7]. A contraction of the hyperedge $e$ is to weakly delete $e$ from $H$ and replace all vertices of $e$ by one vertex belonging to each $e^{\prime}$ such that $e^{\prime} \cap e \neq \emptyset$.

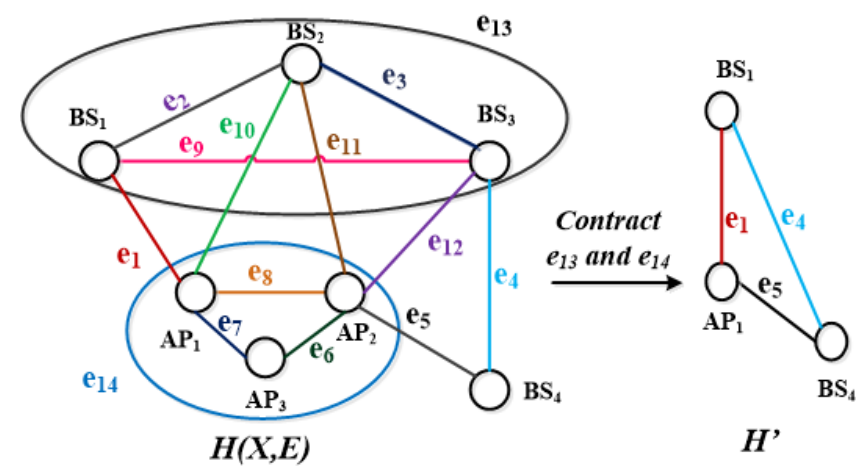

Fig. 2: An example of hypergraph modelling.

\section{A. Hypergraph Construction and Hyperedge Contraction}

An example of hypergraph modelling is given in Fig. 2. Edges $e_{1}$ to $e_{12}$ and $e_{13}$ to $e_{14}$ represent interference and spectral coexistence relations, respectively.

The first step is to construct the edges that represent pairwise interference. Next, spectral coexistence analysis is performed and the information from the coexistence matrix is then processed to generate "interference coordination subsets", such that each node in the subset can coexist with every other node in the subset, and the total COR of the elements of the subset is $\leq$ unity. Interference coordination subsets form the hyperedges. To reduce complexity, intersection of hyperedges is avoided. The procedure for hyperedge construction is summarised in Algorithm 3.

Hyperedge contraction theory is then applied to decompose the hypergraph $H$ into the form of a traditional graph and make it amenable to the vertex colouring algorithm, without losing coexistence information. The output is a minor graph $H^{\prime}$ in which each hyperedge is contracted to a single vertex, such that colouring of that single vertex implies assigning a channel to a group of coexistent networks.

\section{B. Channel Allocation Algorithm}

This involves applying the vertex colouring algorithm to the minor graph and then applying the resultant colour map to the hypergraph, while ensuring that the vertices of a hyperedge get the colour that is assigned to the vertex of the minor graph that replaced all the vertices of that hyperedge. This is illustrated in Fig. 3.

However, it was noted during algorithm testing that applying the vertex colouring algorithm to the minor graph may in some cases not exhaust all possible channel allocations. In hyperedge contraction, all vertices in a hyperedge are replaced by a single vertex such that all edges that were incident on the individual vertices are now incident on the new vertex, hence information about some possible channel allocations could be masked. For instance, in Fig. 3, when hyperedge $e_{13}$ is contracted, there will be an edge between the new vertex $B S_{1}$ and $B S_{4}$ in $H^{\prime}$. However, it can be noted in $H$ that $B S_{4}$ can share the same channel with $B S_{3}$ if they are able to coexist and calculation of total COR will only involve $B S_{3}$ 

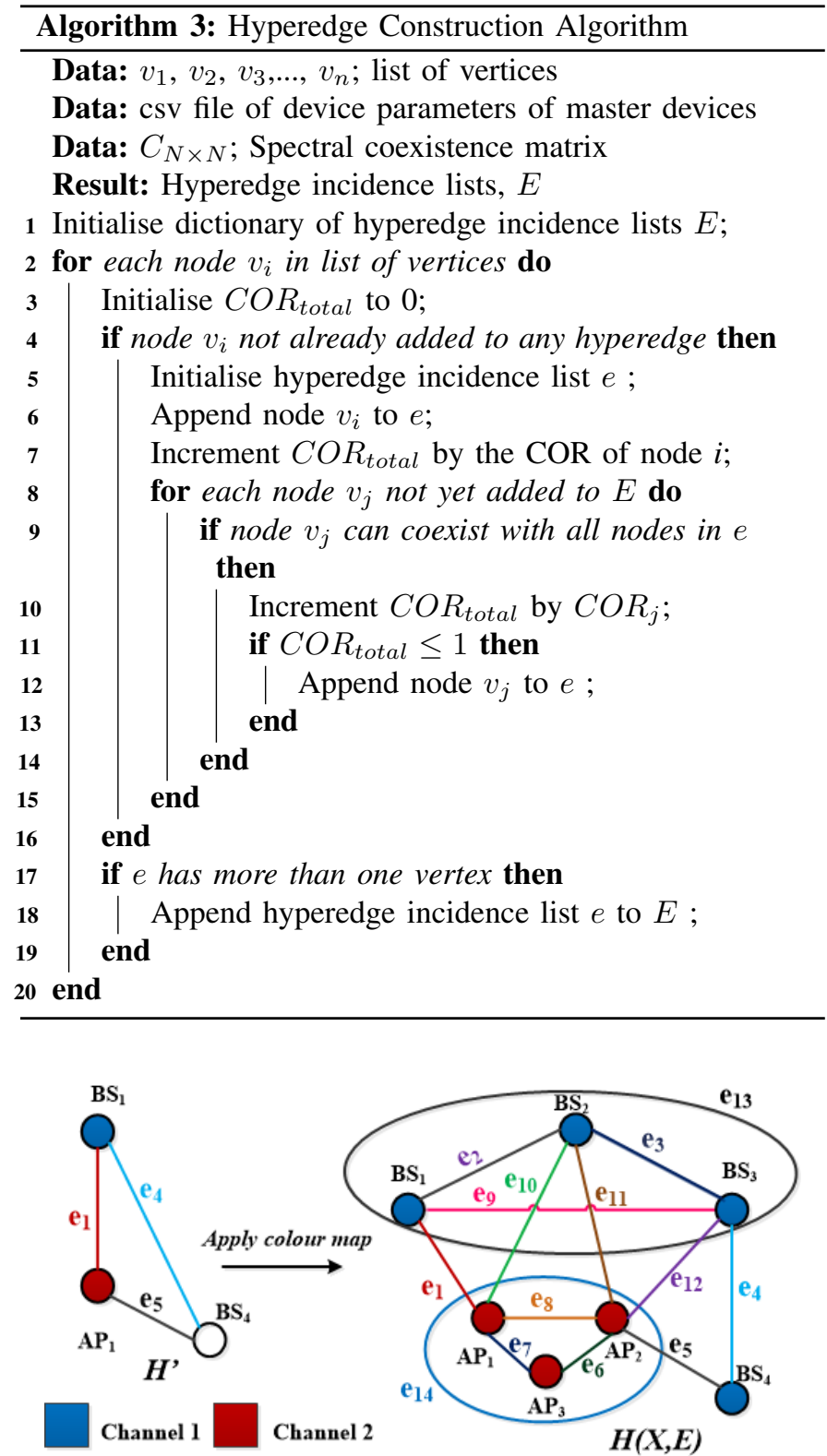

Fig. 3: An example of hypergraph colouring.

and $B S_{4}$ because $B S_{4}$ is not visible to $B S_{1}$ and $B S_{2}$. Hence, the algorithm was augmented to check for additional possible allocations that do not violate interference, coexistence and COR constraints if there are still some uncoloured nodes after vertex colouring of the minor graph. The entire procedure is summarised in Algorithm 4.

\section{Simulation Results}

A $50 \mathrm{~km}$ by $50 \mathrm{~km}$ test area centred around Lilongwe city in Malawi is considered. The geo-locations of the networks are randomly and independently generated within the test area. The rest of the test parameters are given in Table I. The following propagation model, which is suggested in the IEEE 802.19.1 standard, is used to predict the path loss of the transmissions: $L(d)=10 \log \left(\frac{4 \pi d}{\lambda}\right) \alpha-20 \log \left(h_{t} * h_{r}\right)$.

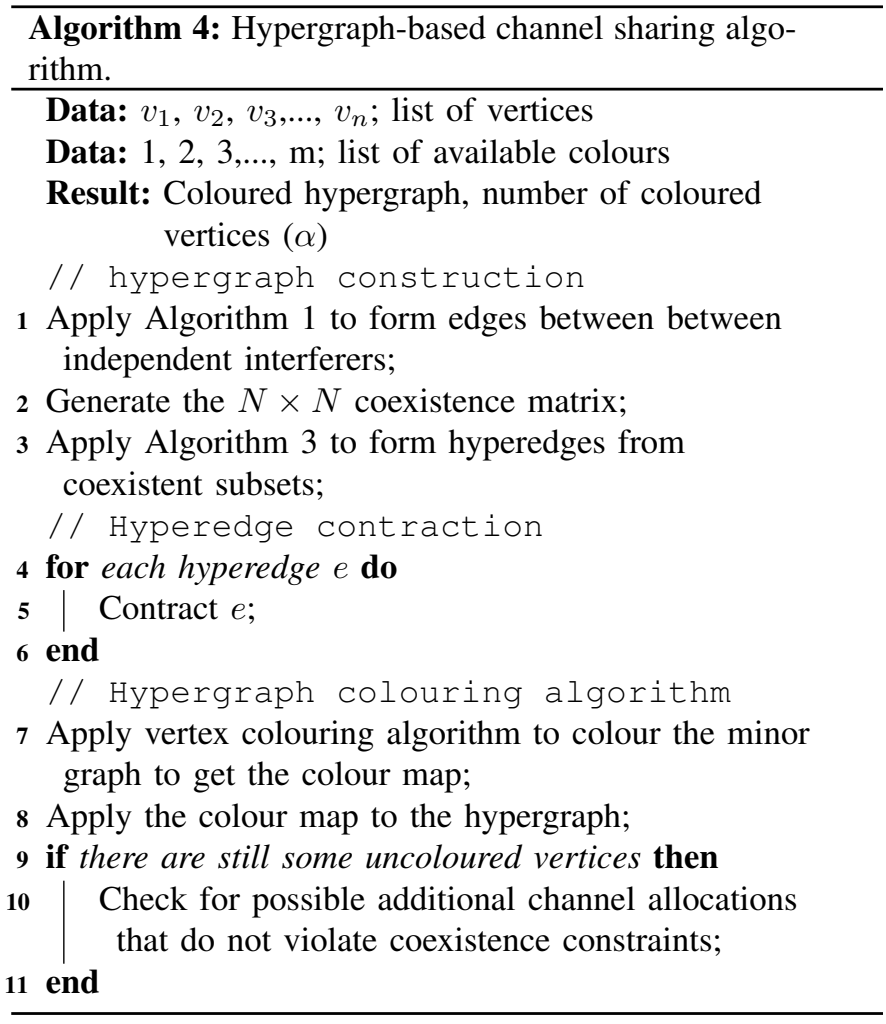

The Python programming language was used to simulate the channel allocation algorithms in order to ascertain that the proposed hypergraph model enables more efficient spectrum usage than the traditional graph model.

Fig. 4 presents the distribution of the results, from 100 simulations, for the number of operational networks $(\alpha)$ as a function of the total number of competing networks $(N)$. It is assumed that there are initially 50 networks of $1 \mathrm{~km}$ radius in the test area. Then 50 networks of $10 \mathrm{~km}$-radius are added, five at a time. From $N=55$, when the $10 \mathrm{~km}$ radius networks start to join, some $1 \mathrm{~km}$-radius networks start to lose their operating channels to the $10 \mathrm{~km}$-radius network because vertices of the long-range networks are likely to have a higher vertex degree in the interference graph. As more $10 \mathrm{~km}$-radius networks join, they share operating channels

TABLE I: Parameters for Simulation

\begin{tabular}{|l|l||l|}
\hline Radio Access Technology (RAT) & $\begin{array}{l}802.11 \\
\text { TVHT }\end{array}$ & $\begin{array}{l}802.22 \\
\text { WRAN }\end{array}$ \\
\hline \hline Cell coverage $(\mathrm{m})$ & 1,000 & 10,000 \\
\hline AP/BS conducted power $(\mathrm{dBm})$ & 15 & 31 \\
\hline AP/BS antenna gain $(\mathrm{dB})$ & 5 & 5 \\
\hline UE/CPE antenna gain $(\mathrm{dB})$ & 0 & 11 \\
\hline AP/BS antenna height $(\mathrm{m})$ & 10 & 15 \\
\hline UE/CPE antenna height $(\mathrm{m})$ & 1.5 & 10 \\
\hline Attenuation factor, $\alpha$ & 4 & 4 \\
\hline Channel Occupancy Rate $(\mathrm{COR})$ & 0.3 & 0.25 \\
\hline Centre frequency $(\mathrm{MHz})$ & 474 & 474 \\
\hline SINR threshold $(\mathrm{dB})$ & 10 & 10 \\
\hline
\end{tabular}




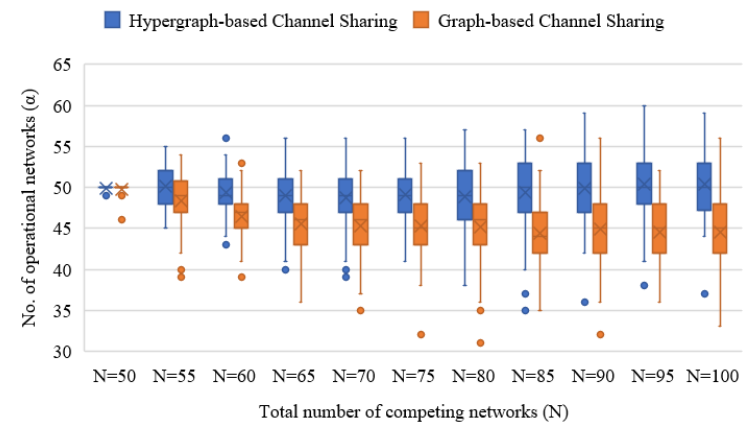

(a) $M=2$

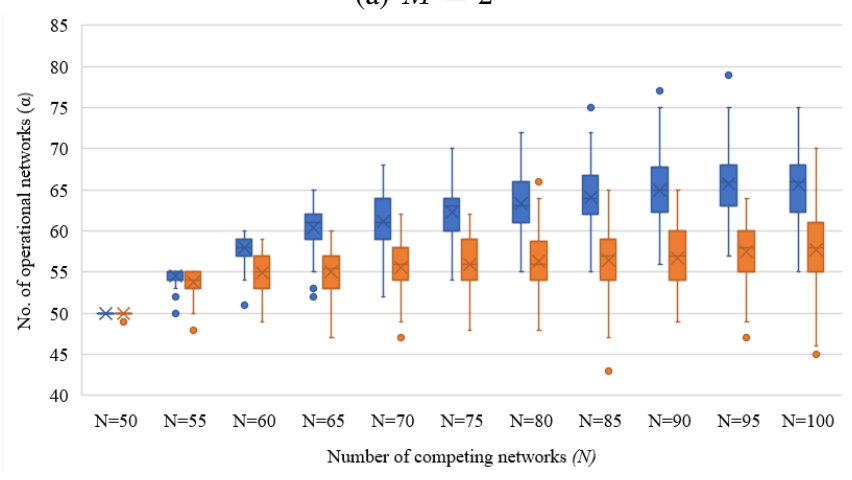

(b) $M=3$

Fig. 4: Average number of operational networks as the number of competing networks is increased.

among them. When $N=100$ the hypergraph-based model achieves average number of operational networks that is $6 \%$ and $8 \%$ higher than in the graph-based model when $M=2$ and $M=3$, respectively, because of the efficient modelling of coexistence information for channel sharing.

The distribution for results of the number of operational networks $(\alpha)$ as a function of the total number of available channels $(M)$, from 20 simulations, is presented in Fig. 5. The number of competing networks is held constant at $N=100$, while the number of available channels is increased steadily from $M=4$. Whereas the graph-based model requires 14 channels to achieve. on average, $100 \%$ operational networks, the hypergraph model requires only 9 . These results imply that, although both models use the same vertex colouring algorithm, the hypergraph model enables the minor graph to be colourable by fewer colours than the traditional graph.

\section{CONCLUSION}

In this paper, hypergraph theory is presented for modelling radio resource allocation that enables coexistence and channel load management in co-channel sharing. Simulation results show that the hypergraph-based model increases the average spectrum utilisation by up to $8 \%$ and reduces the number of channels required to achieve, on average, $100 \%$ operational networks by up to 5 channels. The hypergraph-based model enables more efficient spectrum utilisation than the graphbased model because, unlike in the graph model where spectrum sharing is limited by the outcome of the initial exclusive

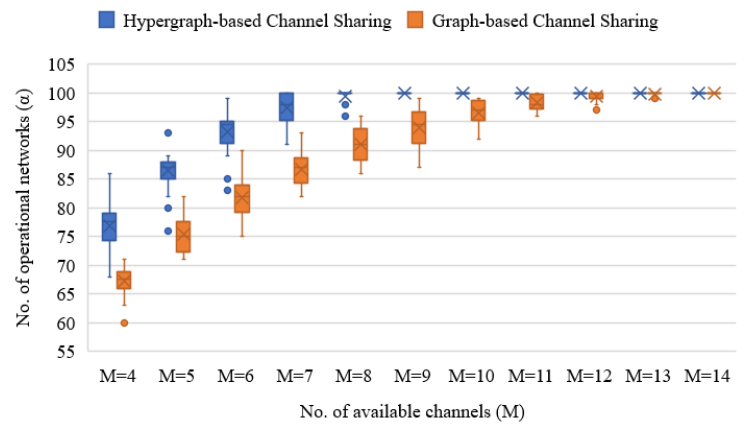

Fig. 5: Number of channels required to achieve, on average, $100 \%$ operational networks

channel allocation, information about spectral coexistence is represented in the hypergraph model before any channel allocation algorithm is applied. Future work shall investigate application of the model to coexistence management among GAA user networks in CBRS.

\section{ACKNOWLEDGMENT}

T.N. thanks the UK government, the Schlumberger Foundation and the University of Strathclyde for funding her studentship.

\section{REFERENCES}

[1] T. Nyasulu, D. H. Crawford, and C. Mikeka, "Malawi's tv white space regulations:: A review and comparison with fcc and ofcom regulations," in 2018 IEEE Wireless Communications and Networking Conference (WCNC), 2018, pp. 1-6.

[2] S. Sagari, I. Seskar, and D. Raychaudhuri, "Modeling the coexistence of lte and wifi heterogeneous networks in dense deployment scenarios," in 2015 IEEE International Conference on Communication Workshop (ICCW), 2015, pp. 2301-2306.

[3] O. Karatalay, S. Erküçük, and T. Baykaş, "Analysis of extended busy tone performance for coexistence between wran and wlan tvws networks," in 2015 IEEE 26th Annual International Symposium on Personal, Indoor, and Mobile Radio Communications (PIMRC), Aug 2015, pp. 1957-1962.

[4] J. Wang, T. Baykas, S. Filin, M. A. Rahman, C. Song, and H. Harada, "Coexistence protocol design for autonomous decision-making systems in tv white space," in 2012 IEEE Wireless Communications and Networking Conference (WCNC), April 2012, pp. 3249-3254.

[5] W. Gao and A. Sahoo, "Performance impact of coexistence groups in a gaa-gaa coexistence scheme in the cbrs band," IEEE Transactions on Cognitive Communications and Networking, vol. 7, no. 1, pp. 184-196, 2021.

[6] X. Ying, M. M. Buddhikot, and S. Roy, "Sas-assisted coexistenceaware dynamic channel assignment in cbrs band," IEEE Transactions on Wireless Communications, vol. 17, no. 9, pp. 6307-6320, 2018.

[7] V. Voloshin, Introduction to Graph and Hypergraph Theory. New York: Nova Science Publishers, Incorporated, 2009.

[8] B. Wang, R. Zhang, C. Chen, X. Cheng, and L. Yang, "Interference hypergraph-based resource allocation (ihg-ra) for noma-integrated $\mathrm{v} 2 \mathrm{x}$ networks," in 2018 IEEE Global Communications Conference (GLOBECOM), 2018, pp. 1-6.

[9] G. Nychis, C. Tsourakakis, S. Seshan, and P. Steenkiste, "Centralized, measurement-based, spectrum management for environments with heterogeneous wireless networks," in 2014 IEEE International Symposium on Dynamic Spectrum Access Networks (DYSPAN), April 2014, pp. $303-$ 314.

[10] C. Sum, M. Zhou, L. Lu, F. Kojima, and H. Harada, "Performance and coexistence analysis of multiple ieee $802 \mathrm{wpan} / \mathrm{wlan} / \mathrm{wran}$ systems operating in tv white space," in 2014 IEEE International Symposium on Dynamic Spectrum Access Networks (DYSPAN), April 2014, pp. 145148. 